MODELING, IDENTIFICATION AND CONTROL, 1994, VOL. 15, NO. 2, 93-107

doi:10.4173/mic.1994.2.3

\title{
Improved independent design of robust decentralized controllers
}

\author{
MORTEN HOVD $†$ and SIGURD SKOGESTAD $† \S$
}

Keywords: Decentralized control; internal model control; structured singular value

The procedure for independent design of robust decentralized controllers proposed by Skogestad and Morari is improved by requiring that each individual controller is of the internal model control (IMC) type. It is shown how to find bounds on the magnitude of the IMC filter time constants such that robust stability or performance is guaranteed. In contrast, Skogestad and Morari found bounds on the sensitivity functions and complementary sensitivity functions for the individual loops, and therefore allowed a much larger class of designs, resulting in more conservative conditions. A property called robust decentralized detunability (RDD) is introduccd. If a system has this property, any subset of the loops can be detuned independently and to an arbitrary degree without endangering robust stability. A simple test for RDD is developed for systems controlled by decentralized IMC controllers.

\section{Introduction}

Decentralized control remains popular in the chemical process industry, despite developments in advanced controller synthesis procedures leading to full multivariable controllers. Some of the reasons for the continued popularity of decentralized control are

(1) Decentralized controllers are easy to implement.

(2) They are easy for operators to understand.

(3) The operators can be allowed to retune the controllers to take account of changing process conditions (as a result of (2) above).

(4) Some measurements of manipulated variables may fail. Tolerance of such failures is more easily incorporated into the design of decentralized controllers than full controllers.

(5) The control system can be brought gradually into service during process startup and taken gradually out of service during shutdown.

A system is said to have robust stability if it is stable regardless of whatever uncertainty is contained within the system. Because of items (4) and (5) above, we would like the system to remain stable if any subset of the control loops is out of service, or if the individual controllers have been detuned. Furthermore, we would like this stability to be a robust property. We define such systems to be robust decentralized detunable

Received 15 December 1992.

† Chemical Engineering, University of Trondheim, NTH, N-7034 Trondheim, Norway.

\$Present address: Fantoft Prosess A/S, PO Box 306, N-1301 Sandvika, Norway.

$\S$ To whom correspondence should be addressed.

(C) 1993 Butterworth-Heinemann Ltd. Reprinted from J. Proc. Cont., 1993, Vol. 3, No. 1, pp. 43-51, with permission of Butterworth-Heinemann Ltd. 
Definition 1: A closed loop system is said to be robust decentralized detunable (RDD) if each controller element can be detuned independently by an arbitrary amount without endangering robust stability.

Decentralized detunability for a given controller should not be confused with decentralized integral controllability (DIC), which is a property of the plant only. DIC implies that there exists for a given plant a decentralized controller with integral action in all channels that is decentralized detunable. Note that the notion of RDD requires that for each controller parameterization one defines more precisely what is meant by 'detuning'. For a PID controller, detuning usually refers to reducing the gain, increasing the integral time or decreasing the derivative time (or all combined). For an IMC controller detuning refers to increasing the filter time constant, $\boldsymbol{\epsilon}_{i}$.

The design of a decentralized control system consists of two main steps

(A) Control structure selection, that is, choosing manipulated inputs and controlled outputs, and pairing inputs and outputs.

(B) Design of each single-input single-output (SISO) controller.

In this paper we will consider step B, and assume that step $A$ has already been done (e.g. by using the tools in Hovd and Skogestad (1992a, b)).

Standard controller synthesis algorithms (e.g. $\mathrm{H}_{2}$ or $\mathrm{H}_{\infty}$ synthesis) lead to multivariable controllers, and cannot handle requirements for controllers with a specified structure. Instead, some practical approaches to the design of decentralized controllers have evolved

- Parameter optimization

- Sequential design (Mayne 1973, Nett and Uthgenannt 1988, Chiu and Arkun 1992).

- Independent design (Skogestad and Morari 1989, Hord and Skogestad 1992c, Braatz, Morai and Skogestad 1992).

In this work we consider independent design, and throughout we will use the structured singular value (see below) as the measure of control quality.

The independent design procedure presented in this paper consists of three key steps

(1) Treat the controller parameters as real design uncertainty.

(2) Ensure that only parameters with the correct sign are allowed by the design uncertainty description.

(3) Find bounds on the controller parameters such that robust stability or robust performance is guaranteed.

In this paper this procedure is applied to an IMC type controller, but a similar procedure may be applied to other controller parameterizations.

\section{Notation}

In this paper, $G(s)$, with elements $g_{i j}(s)$, will denote the plant, which is assumed to be of dimensions $n \times n$. The matrix consisting of the diagonal elements of $G(s)$ is denoted $\tilde{\boldsymbol{G}}(s)=\operatorname{diag}\left\{g_{i j}(s)\right\}$. The reference signal (set-point) is denoted $r$, manipulated inputs are denoted $u$ and outputs $y$. If disturbances are present, $G_{d}(s)$ denotes the (open loop) transfer function from disturbances $d$ to outputs $y$. Throughout this work, all 
(a)

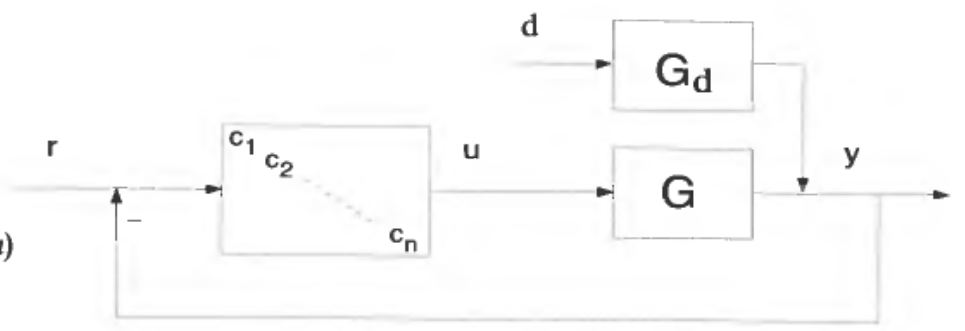

(b)

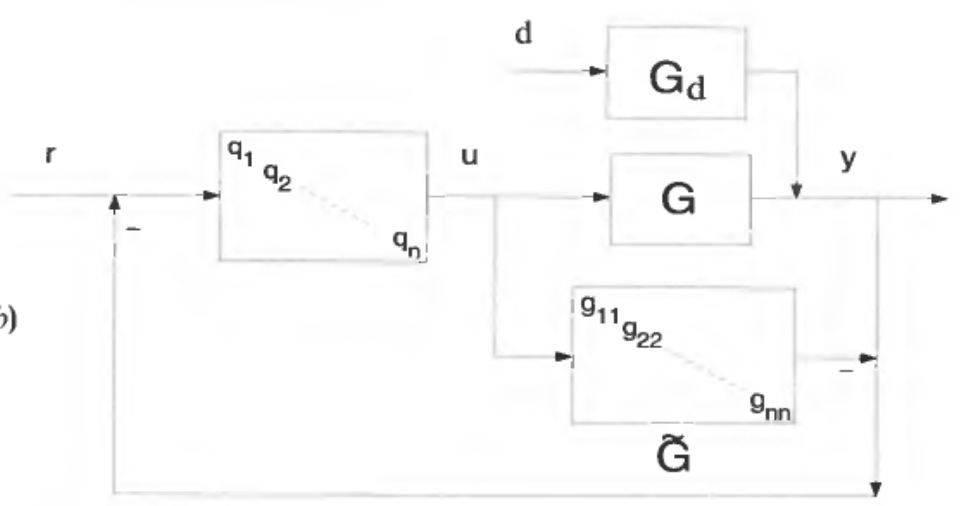

Figure 1. Block diagram of feedback systems: (a) conventional decentralized controller; (b) decentralized IMC controller.

controllers are assumed to be completely decentralized. The decentralized conventional feedback controller is denoted $C(s)$, with ith diagonal element $c_{i}(s)$ (Fig. $1(a)$ ). Likewise, the decentralized IMC controller is denoted $Q$, with ith diagonal element $q_{i}(s)$ (Fig. $1(b)$ ).

The sensitivity function is $S(s)=(I+G(s) C(s))^{-1}$ the complementary sensitivity function is $H(s)=I-S(s)=G(s) C(s)(I+G(s) C(s))^{-1}$. The sensitivity functions and complementary sensitivity functions for the individual loops are collected in the diagonal matrices $\widetilde{\boldsymbol{S}}(s)=(I+\widetilde{G}(s) C(s))^{-1}$ and $\tilde{\boldsymbol{H}}(s)=\widetilde{G}(s) C(s)(I+\widetilde{G}(s) C(s))^{-1}$. Note that the diagonal elements of $\tilde{\boldsymbol{S}}(s)$ and $\tilde{\boldsymbol{H}}(s)$ do not equal the diagonal elements of $S(s)$ and $H(s)$, respectively. The $i$ th elements on the diagonal of $\tilde{S}$ and $\tilde{H}$ are $\tilde{S}_{i}$ and $\tilde{h_{i}}$, respectively.

\section{Robust control and the structured singular value}

Readers familiar with the structured singular value can skip this section. Since no model is a perfect representation of the system, the control system stability and performance should be little affected by the uncertainties of the model. In this paper we use the structured singular value, $\mu$, introduced by Doyle, Wall and Stein (1982), as a measure of the robustness of feedback systems. Within the $\mu$ framework, one accepts that it is impossible to find a perfect model, and instead one requires information about the structure, location and estimates of the magnitude of the model uncertainties.

In Fig. 2 we have drawn an example of a feedback system with uncertainty in the inputs and outputst, represented by the perturbation blocks $\Delta_{\mathrm{I}}$ and $\Delta_{\mathrm{p}}$, respectively.

† Many other types of uncertainties are possible; see Doyle et al. (1982) for details on how to represent different uncertainties with perturbation blocks. 


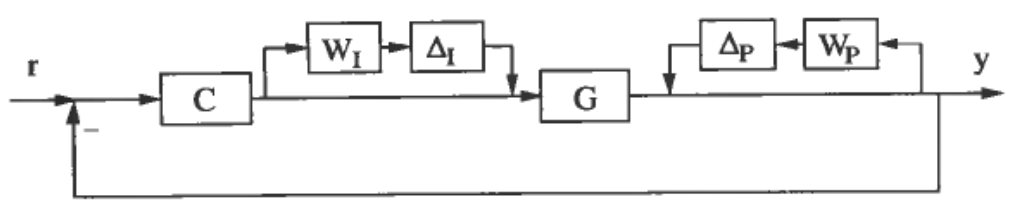

Figure 2. Block diagram for feedback system with multiplicative input uncertainty and inverse multiplicative output uncertainty. The inverse uncertainty may alternatively represent a performance specification in terms of weighted sensitivity.

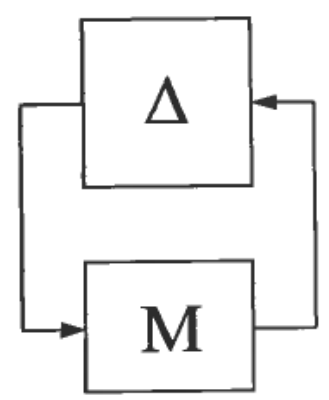

Figure 3. Feedback system rearranged into a perturbation block $\Delta$ and an interconnection matrix $\boldsymbol{M}$.

Note that the individual perturbations can be restricted to have a certain structure. For instance, as individual inputs and outputs usually do not affect each other, both $\Delta_{I}$ and $\Delta_{\mathrm{P}}$ may be diagonal. The weights $W_{1}$ and $W_{\mathrm{P}}$ are frequency-dependent and normalize the maximum magnitude of $\Delta_{\mathrm{I}}$ and $\Delta_{\mathrm{P}}$ to unity.

Any block diagram with uncertainties represented by perturbation blocks can be rearranged into the $M-\Delta$ structure of Fig. 3, if external inputs and outputs are neglected. In Fig. 3, $\Delta$ is a block diagonal matrix with the perturbation blocks of the original block diagram on the diagonal, and $M$ contains all the other blocks in the block diagram (plant, controller, weights). For the specific case in Fig. 2, we have that:

$$
\begin{gathered}
\Delta=\operatorname{diag}\left\{\Delta_{\mathrm{I}}, \Delta_{\mathrm{p}}\right\} \\
\boldsymbol{M}=\left[\begin{array}{cc}
-W_{1} C G(I+C G)^{-1} & -W_{\mathrm{l}} C(I+G C)^{-1} \\
\left.W_{\mathrm{p}} G(I+C G)^{-1}\right) & W_{\mathrm{p}}(I+G C)^{-1}
\end{array}\right]
\end{gathered}
$$

Provided $M$ is stable (the system has nominal stability, NS) and $\Delta$ is norm bounded and stable (stable perturbation blocks), it follows from the Nyquist stability criterion (Doyle et al. 1982) that the overall system is stable provided $\operatorname{det}(I-M \Lambda) \neq 0 \forall \Delta, \forall \omega$. In this case the system is said to have robust stability (RS). The structured singular value is defined such that:

$$
\mu_{\Delta}^{-1}=\min \{\delta \mid \operatorname{det}(I-M \Delta)=0 \text { for some } \Delta, \bar{\sigma}(\Delta) \leqslant \delta\}
$$

If weights are used to normalize the maximum value of the largest singular value of $\Delta$ to unity $(\bar{\sigma}(\Delta)=1$ ) at all frequencies, as in Fig. 2, the system is robustly stable (RS) for any allowable perturbation $\Delta$ provided $\mu_{\Delta}(M)<1$.

Doyle (1982) showed that performance can be analysed in the $\mu$ framework by considering an equivalent stability problem of larger dimension. In this paper we use a performance specification of the type $\bar{\sigma}\left(W_{\mathrm{p}} S_{\mathrm{p}}\right) \leqslant 1 \forall \omega$ where $S_{\mathrm{p}}$ is the worst sensitivity 
function $(S)$ made possible by the perturbation blocks. This can be represented in Fig. 2 by letting the block $\Delta_{\mathrm{p}}$ be a full matrix which represents the performance specification $\bar{\sigma}\left(W_{\mathrm{p}} S_{\mathrm{p}}\right) \leqslant 1$ rather than uncertainty, and robust performance (RP) may be tested in the same way as robust stability. To simplify the notation, we will in some cases use $\mu(M)$ to mean $\sup _{\omega} \mu_{\Delta}(M)$. Doyle and Chu (1985) proposed an algorithm for the synthesis of controllers which minimizes $\mu$, known as $D-K$ iteration. However, $D-K$ iteration results in full controllers, and the problem of synthesizing $\mu$-optimal decentralized controllers has not been solved. This paper discussed a procedure for independently designing decentralized controllers which yield $\mu(M) \leqslant 1$.

\section{Independent design}

Independent design of robust decentralized controllers was introduced by Skogestad and Morari (1989). It is based on Theorem 1 in Skogestad and Morari (1988), which we restate here.

Theorem 1: Let the $\mu$ interconnection matrix $\boldsymbol{M}$ be written as lower linear fractional transformation (LFT) of the transfer function matrix $T$

$$
\boldsymbol{M}=F_{1}(N, T)=N_{11}+N_{12} T\left(I-N_{22} T\right)^{-1} N_{21}
$$

Assume $\mu_{\Delta}\left(N_{11}\right)<1$ and $\operatorname{det}\left(I-N_{22} T\right) \neq 0$, then

$$
\mu_{\Delta}(M) \leqslant 1
$$

if

$$
\bar{\sigma}(T) \leqslant c_{T}
$$

where $c_{T}$ solves

$$
\begin{gathered}
\boldsymbol{\mu}_{\hat{\Delta}}\left[\begin{array}{cc}
N_{11} & N_{12} \\
c_{T} N_{21} & c_{T} N_{22}
\end{array}\right]=1 \\
\text { and } \hat{\Delta}=\operatorname{diag}\{\Delta, T\}
\end{gathered}
$$

Proof: See Skogestad and Morari (1988).

The condition $\mu_{\Delta}(M) \leqslant 1$ is typically the RP condition we want to satisfy, and $T$ is some important transfer function which depends on the controller. $c_{T}$ represents the upper bound on the design 'uncertainty' allowed for $\boldsymbol{T}$. Skogestad and Morari (1989) use Theorem 1 to find bounds on the sensitivity function and complementary sensitivity functions for the individual loops (i.e. $\boldsymbol{T}=\tilde{\boldsymbol{S}}$ and $\boldsymbol{T}=\tilde{\boldsymbol{H}}$ are used). The bounds on $\tilde{S}$ and $\tilde{H}$ can be combined over different frequency ranges. Thus, if either the bound on $\tilde{\boldsymbol{S}}$ or the bound on $\tilde{\boldsymbol{H}}$ is fulfilled for all loops at all frequencies, then $\mu_{\Delta}(M) \leqslant 1$ is achieved.

The rationale behind Theorem 1 is to treat the transfer functions $(T)$ as a 'class of possible designs' (i.e. as design uncertainty), and find bounds on the magnitude of this fictitious uncertainty which guarantee that $\mu_{\Delta}(M) \leqslant 1$. One is subsequently faced finding controllers such that the bounds on the transfer functions are fulfilled. It is therefore important for the success of independent design that $\boldsymbol{T}$ introduces as little additional uncertainty as possible. It turns out that parameterizing the class of possible designs as $\boldsymbol{T}=\tilde{\boldsymbol{S}}$ and $\boldsymbol{T}=\tilde{\boldsymbol{H}}$ is not ideal for this purpose. 
Example 1

Consider Example 1 in Chiu and Arkun (1992)

$$
\boldsymbol{G}(\mathbf{s})=\left[\begin{array}{l}
\frac{1 \cdot 66}{39 s+1} \frac{-1 \cdot 74 e^{-2 s}}{4 \cdot 4 s+1} \\
\frac{0 \cdot 34 e^{-s}}{8 \cdot 9 s+1} \frac{1 \cdot 4 e^{-s}}{3 \cdot 8 s+1}
\end{array}\right]
$$

There is independent input uncertainty with input uncertainty weight $W_{1}(s)=0.07 I_{2}$, and the performance requirement is given by the performance weight $W_{\mathrm{p}}(s)=0.25$ $((7 s+1) / 7 s) I_{2}$.

The condition for robust performance becomes $\mu_{\Delta}(M) \leqslant 1$, with $\Delta=\operatorname{diag}\left\{\Delta_{\mathrm{l}}, \Delta_{\mathrm{P}}\right\}$. $\Delta_{\mathrm{I}}$ is a complex diagonal matrix representing the input uncertainty, and $\Delta_{\mathrm{P}}$ is a full complex matrix representing the performance. The interconnection matrix $\boldsymbol{M}$ is given in Equation (2). Chiu and Arkun (1992) attempted independent design for this example, using $\boldsymbol{T}=\tilde{\boldsymbol{S}}$ and $\boldsymbol{T}=\tilde{\boldsymbol{H}}$, but were unable to find an IMC controller which fulfilled the resulting bounds. We will, however, demonstrate that independent design can be used to design an IMC controller for this example, if we parameterize the class of possible designs in terms of the IMC filter time constant $\epsilon_{i}$ rather than in terms of $\widetilde{\boldsymbol{S}}$ and $\tilde{\boldsymbol{H}}$.

\subsection{Independent design with decentralized IMC controllers}

We use the internal model control (IMC) technique (Garcia and Morari 1982) to parameterize the individual controller elements $c_{i}$, and select $T$ as a parameterization of the tuning constant $\epsilon_{i}$ in the IMC controller. Note that the design of $c_{i}$ is based on the corresponding diagonal element of the plant, and this may not be a good choice in some cases. Our approach is similar to that of Lee and Morari (1991), but we use $\epsilon_{i}$ as the parameter rather than the filter $f_{i}$. The relationship between the elements $q_{i}$ of the IMC controller and the elements $c_{i}$ of the conventional decentralized controller is given by (Fig. 1):

$$
\epsilon_{i}=q_{i}\left(1-g_{i i} q_{i}\right)^{-1}
$$

In the IMC design procedure $q_{i}$ has the form:

$$
q_{i}=\hat{g}_{i i}^{-1} f_{i}
$$

where $\hat{g}_{i i}$ is the minimum phase part of $g_{i i}$, and $f_{i}$ is a low-pass filter used to make $q_{i}$ realizable and to detune the system for robustness. In order to simplify the exposition, we will assume the plant $G$ to be open loop stable, and use a low-pass filter of the form:

$$
f_{i}=\frac{1}{\left(\epsilon_{i} s+1\right)^{n f}}
$$

That is, the $f_{i}$ is taken to be a low-pass filter of order $n_{f}$, consisting of $n_{f}$ identical firstorder low-pass filters in series.

Choice of $\mathrm{T}$ for independent design. After fixing $n_{f}$, the only thing which remains unknown in the IMC technique is the value of $\epsilon_{i}$. To fulfil performance requirements at low frequencies, the closed loop system must be sufficiently fast, which means that the filter time constant $\epsilon_{i}$ must be smaller than a certain value. On the other hand, the closed loop system must be sufficiently detuned to avoid robustness problems at higher 


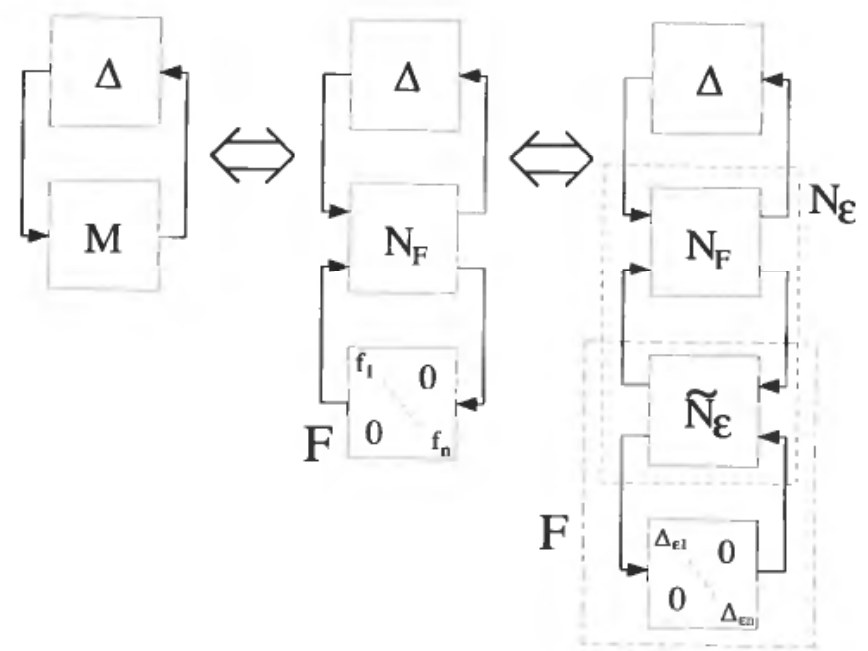

Figure 4. The interconnection matrix $\boldsymbol{M}$ expressed as an LFT of the IMC filter $\boldsymbol{F}$ and as an LFT of the 'uncertainty' associated with the filter time constants.

frequencies, thus requiring $c_{i}$ to be larger than a certain value, meaning that $1 / \epsilon_{i}$ must be smaller than some value. We will therefore use Theorem 1 to find bounds on $\epsilon_{i}$ and also on:

$$
e_{i}=1 / \epsilon_{i}
$$

which can be combined over different frequency ranges. Since we are using a specific control structure, the class of possible designs is much smaller than if we use Theorem 1 to find bounds on $\tilde{\boldsymbol{S}}$ and $\tilde{\boldsymbol{H}}$. Bounds on $\tilde{\boldsymbol{S}}$ and $\tilde{\boldsymbol{H}}$ are therefore potentially much more conservative.

To derive conditions on $\epsilon_{i}$ and $e_{i}$ that guarantee $\mu_{\Delta}(M) \leqslant 1$ we will proceed as follows. First we parameterize the $\mu$ interconnection matrix $\boldsymbol{M}$ as an LFT of the IMC filter $F$ and then as an LFT of the 'uncertainty' in the filter time constant. We refer readers to Skogestad and Morari (1988) for details on how to find the matrix $N_{\mathrm{F}}$ needed in Fig. 4. We will only elaborate on how to find $\tilde{N}_{c}$, i.e. to express $f_{i}$ as an LFT of the design 'uncertainty' $T=\Delta$, or $T=\Delta_{e}$ associated with $\epsilon_{i}$ or $e_{i}$. We then solve Eqn (7) at each frequency point to find the desired bound. Note that it is sufficient at each frequency to satisfy the bound either for $\epsilon_{i}$ or for $e_{i}=1 / \epsilon_{i}$, but we must of course use the same bound for $\epsilon_{i}$ (and $e_{i}$ ) at all frequencies. Note that although $\hat{g}_{i i}^{-1}$ in Eqn (10) will normally not be realizable, its frequency response is easily calculated. Also note that since we work with the frequency response, we will have to check $\grave{a}$ posteriori for the (internal) stability of the $\mu$ interconnection matrix.

First-order low-pass filters. Consider first the case $n_{f}=1$. We then have $f_{i}=1 /\left(\epsilon_{i} s+1\right)$. The objective is to find the allowable ranges for $\epsilon_{i}$ and $e_{i} 1 / \epsilon_{i}$ that at each frequency guarantee $\mu(M) \leqslant 1$. Since we do not allow negative values for $\epsilon_{i}$ we should not simply write $\left|\epsilon_{i}\right| \leqslant c_{\epsilon}$. Instead write:

$$
\begin{aligned}
& \epsilon_{i}=\frac{\epsilon^{*}}{2}\left(1+\Delta_{i i}\right)\left|\Delta_{i i}\right| \leqslant 1 \\
& e_{i}=\frac{e^{*}}{2}\left(1+\Delta_{e i}\right)\left|\Delta_{e i}\right| \leqslant 1
\end{aligned}
$$


In order to use Theorem 1 we now need to write $f_{i}$ as an LFT of $\Delta_{i}$ and $\Delta_{e i}$. We have

$$
f_{i}=\frac{1}{\frac{\epsilon^{*}}{2}\left(1+\Delta_{i}\right) s+1}
$$

which may be written as an LFT, $f_{i}=F_{1}\left(\tilde{N}_{i}, \Delta_{i}\right)$, with

$$
\tilde{N}_{c_{i}}=\frac{1}{\frac{\epsilon^{*}}{2} s+1}\left[\begin{array}{cc}
1 & -1 \\
\frac{\epsilon^{*}}{2} s & -\frac{\epsilon^{*}}{2} s
\end{array}\right]
$$

Similarly, $f_{i}=F_{1}\left(\tilde{N}_{e i}, \Delta_{e i}\right)$ with

$$
\tilde{N}_{e_{i}}=\frac{1}{\frac{e^{*}}{2 s+1}}\left[\begin{array}{cc}
e^{*} & e^{*} / 2 \\
s & -e^{*} / a
\end{array}\right]
$$

This shows how to express an individual filter element $f_{i}$ as an LFT of the design 'uncertainty' in the filter time constant $\left(\epsilon_{i}\right.$ or $\left.1 / \epsilon_{i}\right)$. The LFT for the overall IMC filter $F=\operatorname{diag}\left\{f_{i}\right\}$ is then just a simple diagonal augmentation of the corresponding blocks of the LFT for the individual filter elements.

We now use Theorem 1 with $T=\Delta_{c}=\operatorname{diag}\left\{\Delta_{c}\right\}$ and $T=\Delta_{e}=\operatorname{diag}\left\{\Delta_{e i}\right\}$ to derive bounds on $\epsilon_{i}$ and $1 / \epsilon_{i}=e_{i}$, respectively. Using $\left|\Delta_{i}\right| \leqslant 1$ (Eqn (13)) and $\left|\Delta_{e i}\right| \leqslant 1$ (Eqn (14)) corresponds to setting $c_{\mathrm{T}}=1$ in Eqn (7), such that Eqn (7) becomes $\mu_{\vec{\Delta}}(N)=1$, where $\hat{\Delta}=\operatorname{diag}\left\{\Lambda, \Lambda_{c}\right\}$ or $\hat{\Delta}=\operatorname{diag}\left\{\Lambda, \Lambda_{e}\right\} . \Lambda_{c}$ and $\Delta_{e}$ are diagonal matrices with real elements. $N$ depends on $\epsilon^{*}$ or $e^{*}$ (as appropriate), and we denote the values that give $\mu_{\hat{A}}(N)=1$ as $c_{s}^{*}$ and $e_{s}^{*}$, respectively. For a fixed frequency, we are then guaranteed that $\mu_{\Delta}(M) \leqslant 1$ provided

or

$$
\epsilon_{i} \leqslant \epsilon_{s}^{*} \forall i
$$

$$
e_{i} \leqslant e_{s}^{*} \forall i \Leftrightarrow \epsilon_{i} \geqslant \frac{1}{e_{s}^{*}} \forall i
$$

Note that although the $\epsilon_{i}$ s are independent, we get the same bound for all $\epsilon_{i}$.

Note also that $\mu_{\hat{\Delta}}(N)$ is non-decreasing with increasing values of $\epsilon_{s}^{*}$ or $e_{s}^{*}$, such that it is straightforward to obtain $\epsilon^{*}$ and $e^{*}$. This follows from the fact that we use $\left|\Delta_{c}\right| \leqslant 1$ and therefore the set of possible values for $\epsilon_{i}=\left(\epsilon^{*} / 2\right)\left(1+\Delta_{i}\right)$ for any fixed value of $\epsilon^{*}$ contains all the possible values for $\epsilon_{i}$ for any smaller $\epsilon^{*}$ (and similarly for $e_{i}$ and $e^{*}$ ).

Higher order low-pass filters. Above we assumed $n_{f}=1$, but in IMC designs, one will often use filters of order higher than one. We therefore need to be able to express the higher order filters as LFTs of $\Delta_{c}$ and $\Delta_{e}$. For this we can use the rules for series interconnection of linear dynamic systems. First note that $G(s)=C(s I-A)^{-1} B+D$ may be written as an LFT of $(1 / s) I$, with

$$
N_{11}=D ; \quad N_{12}=C ; \quad N_{21}=; \quad N_{22}=A
$$

The formulae for series interconnection $G=G_{1} G_{2}$ of dynamic system $G_{1}(s)=C_{1}$ $\left(s I-A_{1}\right)^{-1} B_{1}+D_{1}$ and $G_{2}(s)=C_{2}\left(s I-A_{2}\right)^{-1} B_{2}+D_{2}$ are (Maciejowski 1989)

$$
\begin{aligned}
\boldsymbol{A} & =\left[\begin{array}{cc}
A_{1} & 0 \\
B_{2} C_{1} & A_{2}
\end{array}\right] \\
\boldsymbol{B} & =\left[\begin{array}{c}
B_{1} \\
B_{2} D_{1}
\end{array}\right]
\end{aligned}
$$




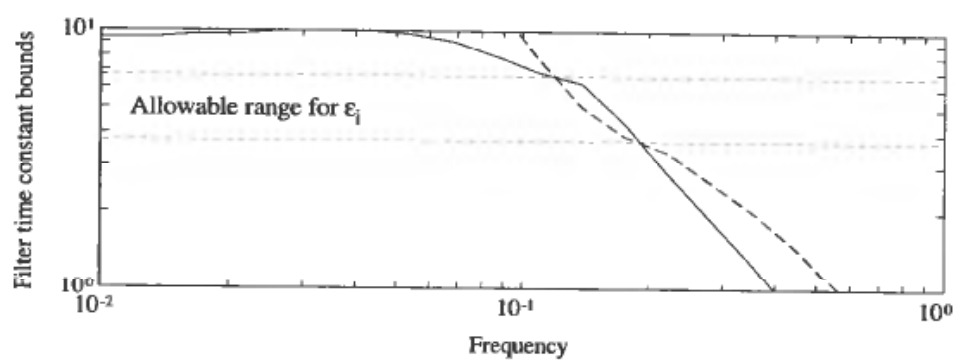

Figure 5. Robust performance bounds on the filter time constant for Example 1. Solid line, $\epsilon_{s}^{*}$ (upper bound); dashed line, $1 / e_{s}^{*}$ (lower bound).

$$
\begin{aligned}
\boldsymbol{C} & =\left[\begin{array}{ll}
D_{2} C_{1} & C_{2}
\end{array}\right] \\
\boldsymbol{D} & =D_{2} D_{1}
\end{aligned}
$$

The formulae for series interconnection of dynamic systems can be used directly to express a low-pass filter element $f_{i}$ of order $n_{f}$ as LFTs of

or

$$
\Delta_{i}=\operatorname{diag}\{\overbrace{\delta_{c i}, \ldots, \delta_{c i}}^{n_{f} \text { times }}\}
$$

$$
\Delta_{e i}=\operatorname{diag}\{\overbrace{\left.\delta_{e i}, \ldots, \delta_{e i}\right\}}^{n_{f} \text { times }}\}
$$

Here we have assumed that the same filter time constant $\epsilon_{i}$ is repeated $n_{f}$ times as given in Eqn (11). Note that the filter time constants $\epsilon_{i}$ in the different filter elements are allowed to differ. For a plant of dimensions $n \times n$ we therefore end up with $n$ repeated scalar uncertainty blocks for the IMC filter, each of these blocks being repeated $n_{f}$ times. $\dagger$

\subsection{Independent IMC design procedure}

With the preliminaries above, we can now propose an independent design algorithm in terms of the IMC filter time constants, $\iota_{i}$ :

Step 1: Find the matrices $\mathrm{N}_{c}$ expressing the $\mu$ interconnection matrix $M$ as an LFT of $\Delta_{\epsilon}$, and the matrix $N_{e}$, expressing M as an LFT of $\Delta_{e}$. $N_{\epsilon}$ will depend on the value of $\epsilon^{*}$, and $N_{e}$ will depend on the value of $e^{*}$, and we must therefore recompute $N_{t}$ and $N_{e}$ for every new value of $\epsilon^{*}$ and $e^{*}$, respectively.

Step 2: We get

if

$$
\mu_{\Delta}(M) \leqslant 1
$$

where $\epsilon_{s}^{*}$ solves:

$$
0 \leqslant c_{i} \leqslant \epsilon_{s}^{*} \forall i
$$

$$
\mu_{\hat{\Delta}}\left(N_{c}\right)=1 \quad \text { where } \hat{\Delta}=\operatorname{diag}\left\{\Delta, \Delta_{c}\right\}
$$

Similarly, let $e_{s}^{*}$ solve $\mu_{\hat{A}}\left(N_{e}\right)=1$, giving the bound

$$
\epsilon_{i} \geqslant 1 / e_{s}^{*} \forall i
$$

$\dagger$ One may use low pass filters of different orders in the different filter elements, in which case the value of $n_{f}$ will differ for different filter elements. 


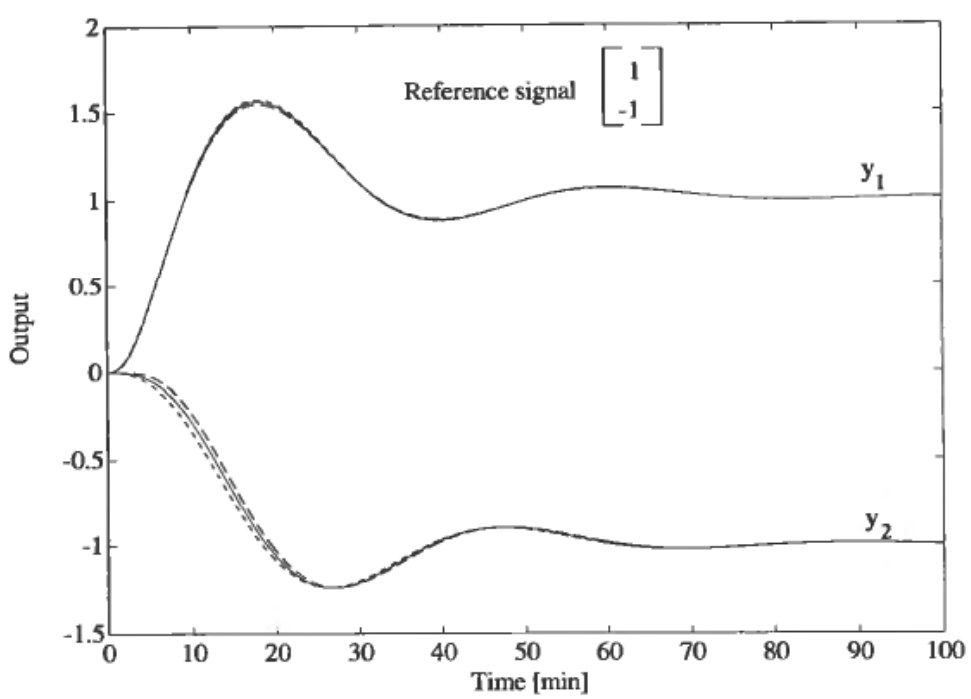

Figure 6. Response for Example 1 using a decentralized IMC controller with second-order filter with filter time constants $\epsilon_{1}=\epsilon_{2}=5 \mathrm{~min}$ in both loops. Solid line, nominal plant; dashed line, $+7 \%$ uncertainty in $u_{1}$ and $-7 \%$ uncertainty in $u_{2}$; dotted line, $-7 \%$ uncertainty in $u_{1}$ and $+7 \%$ uncertainty in $u_{2}$.

Step 3: From Step 2 and Theorem 1 we know that $\mu_{\Delta}(M)<1$ for the range of values of $\epsilon_{i}$ which at all frequencies are either within the range of values in Eqn (20) or within the range of values in Eqn (22).

Step 4: Choose a value of $\epsilon_{i}$ within the range of values found in Step 3, and verify the stability of $M$ for this choice of $c_{i} \uparrow$

If we are successful in Steps 3 and 4, the controller design is completed. Since we have both real and complex perturbations, Step 2 requires $\mu$ calculations for mixed real and complex perturbations (Young, Newlin and Doyle 1991), which is still a research topic. However, the existing $\mu$ software has proved to be acceptable in many cases.

\section{Examples}

Example 1 (continued). Consider again the robust performance problem in Example 1. For this problem we choose a second-order low-pass filter $\left(n_{f}=2\right)$ in each element of the decentralized IMC controller. This filter order is the lowest that gives a strictly proper controller. Since we have a $2 \times 2$ system, this will add two real, repeated scalar perturbations, each repeated twice, i.e. the design uncertainty is $\Delta_{t}=\operatorname{diag}\left\{\delta_{t 1}, \delta_{t 1}, \delta_{t 2}, \delta_{t 2}\right\}$.

From Step 2 in the design procedure, we obtain the results in Fig. 5. We see that values of $\epsilon_{i}$ between 3.7 and 6.6 are at all frequencies either below the upper bound or above the lower bound. Choosing $\epsilon=5$ for both loops, it is easily verified that the system is nominally (internally) stable. We have thus completed an independent design for this example. The response of the system to a step change in the reference signal is shown in Fig. 6 for the choice $\epsilon_{1}=\epsilon_{2}=5$. In Fig. 6 the time delays in the plant are approximated by second order Padé approximations.

+ For any value of $\epsilon_{i}$ within the range found in Step 3, the map under the Nyquist D-contour of $\operatorname{det}(I-M \Delta)$ will encircle the origin the same number of times. Thus, if $M$ is found to be unstable in Step 4, it is 'robustly unstable'. 


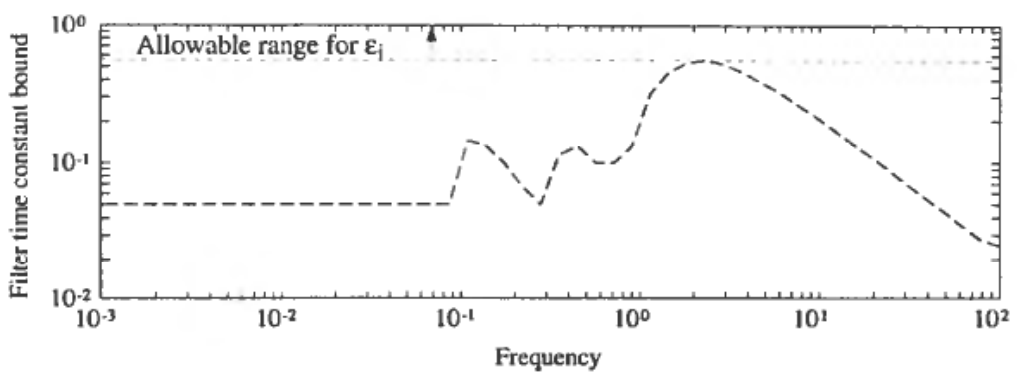

Figure 7. Lower bound on filter time constant $\left(1 / e_{s}^{*}\right)$ for robust stability in Example 2.

Example 2. The following example is provided by Chiu (1991)

$$
\boldsymbol{G}(s)=\left[\begin{array}{ccc}
\frac{0 \cdot 66}{6 \cdot 7 s+1} & \frac{-0 \cdot 61}{8 \cdot 4 s+1} & \frac{-0 \cdot 005}{9 \cdot 06 s+1} \\
\frac{1 \cdot 11}{3 \cdot 25 s+1} & \frac{-2 \cdot 36}{5 s+1} & \frac{-0 \cdot 01}{7 \cdot 09 s+1} \\
\frac{-34 \cdot 7}{8 \cdot 15 s+1} & \frac{46 \cdot 2}{10 \cdot 9 s+1} & \frac{0 \cdot 87(11 \cdot 61 s+1)}{(3 \cdot 89 s+1)(18 \cdot 8 s+1)}
\end{array}\right]
$$

In this example only robust stability is considered, with independent, multiplicative input uncertainty with uncertainty weight $W_{\mathrm{I}}(s)=0-13(5 s+1) /(0 \cdot 25 s+1)$. The interconnection matrix $M=W_{1} C G(I+C G)^{-1}$ and the condition for robust stability is $\mu_{\Delta}(M) \leqslant 1$, where $\Delta$ is a complex diagonal matrix representing the input gain uncertainty. In Chiu (1991) it is found that independent design using Theorem 1 with $\boldsymbol{T}=\tilde{\boldsymbol{H}}$ and $\boldsymbol{T}=\tilde{\boldsymbol{S}}$ cannot be used to design a robust controller for this example. Since the process is stable and only multiplicative uncertainty is considered, this clearly illustrates the shortcomings of that method.

As in Example 1, a second-order low-pass filter is used in each diagonal element of the IMC controller. This will add three real, repeated scalar perturbations, each repeated twice, i.e. the design uncertainty is $\Delta_{\mathrm{t}}=\operatorname{diag}\left\{\delta_{\mathrm{t} 1}, \delta_{\mathrm{t} 1}, \delta_{t 2}, \delta_{t 2}, \delta_{t_{3}}, \delta_{t\}}\right\}$.

From Step 2 in the independent design procedure we obtain the results in Fig. 7. From Fig. 7 we see that any value of $c$ larger than 0.55 will be acceptable. To complete the independent design procedure (Step 4), we choose $\epsilon=1$ for all loops, and find that the system is stable. We thus conclude that the system will be robustly stable for any value of $c_{i}>0.55$.

In general we want $c_{i}$ to be small for a faster nominal responsc. However, since this example contains no performance specification, one should take care when choosing the filter time constants. This is demonstrated in Fig. 8, which shows step responses for the case with large differences in the filter time constants $\left\{\left\{\epsilon_{1}, \epsilon_{2}, \epsilon_{3}\right\}=\{7 \cdot 3,3 \cdot 8,0 \cdot 56\}\right.$, Fig. $8(a))$ and with all loops tightly tuned $\left(\epsilon_{1}=\epsilon_{2}=\epsilon_{3}=0 \cdot 56\right.$, Fig. $\left.8(b)\right)$ both for the nominal case (solid line) and with uncertainty (dashed line). We see that the interactions degrade the performance significantly when all the loops are tightly tuned, even in the nominal case.

Remark: Adding performance requirements and looking for an allowable region for $\epsilon_{i}$ (as we did in Example 1) did not prove successful for Example 2. It thus seems that independent design is not a very favourable design technique for this example, because 


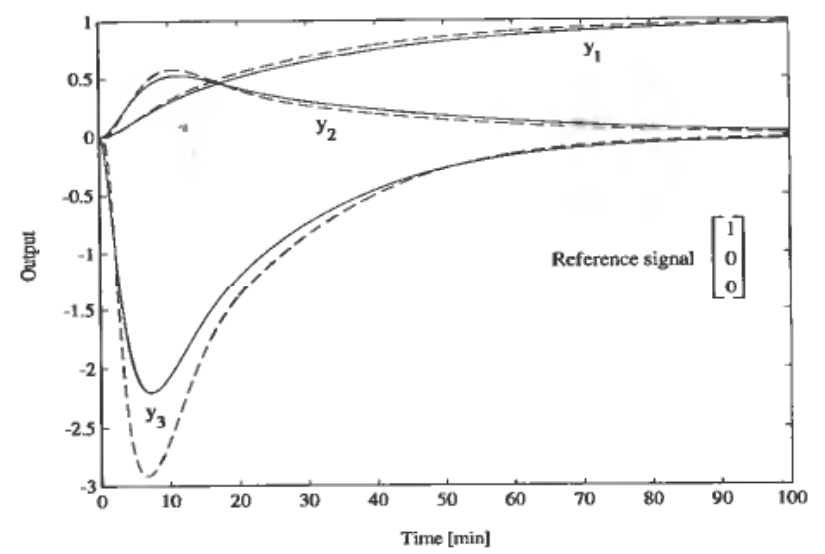

(a)

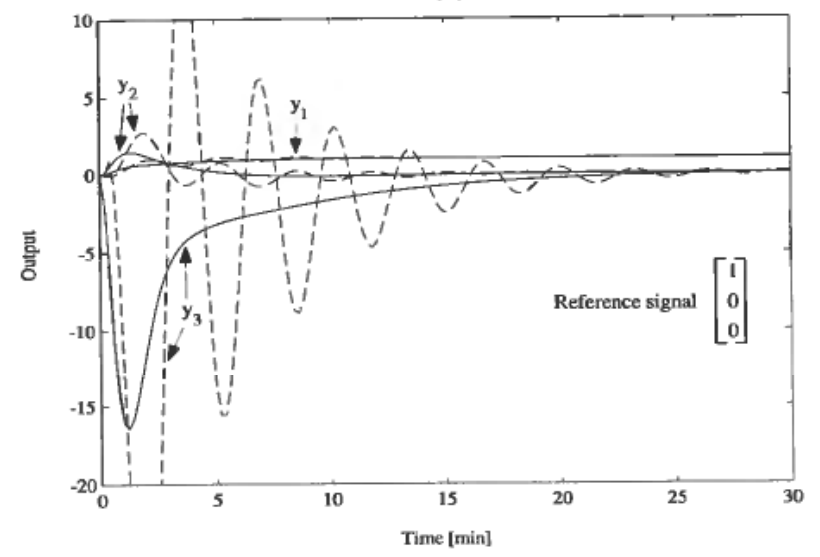

(b)

Figure 8. Responses for Example 2: $(a)\left\{\epsilon_{1}, \epsilon_{2}, \epsilon_{3}\right\}=\{7 \cdot 3,3 \cdot 8,0.56\} ;(b) \epsilon_{1}=\epsilon_{2}=\epsilon_{3}=0.56$. Solid line, nominal plant; dashed line, 0.5 minute time delay in all inputs, $+13 \%$ gain uncertainty in $u_{1}$ and $u_{2}$ and $-13 \%$ gain uncertainty in $u_{3}$.

we cannot take advantage of allowing the loops to be tuned differently. Indeed, the values $\left\{\epsilon_{1}, \epsilon_{2}, \epsilon_{3}\right\}=\{7 \cdot 3,3 \cdot 8,0 \cdot 56\}$ used in Fig. $8(a)$ were based on experience gained using sequential design.

\subsection{Robust decentralized detunability in the IMC framework}

In the IMC framework, controllers are detuned by increasing the filter time constants. We have thus found for Example 2 that the loops can be detuned independently of each other, without endangering robust stability, provided all loops have $\epsilon_{i}>0.55$. Thus the closed loop system in Example 2 with $\epsilon_{i}>0.55$ in all loops is found to be robust decentralized detunable according to Definition 1. Similarly, after removing the performance requirement from Example 1 and redoing the calculations for robust stability, we find that it is robust decentralized detunable provided $c_{i}>0 \cdot 16$ for both loops.

A requirement for robust decentralized detunability is that the individual loops are stable. A decentralized IMC controller as parameterized in Eqn (10) will make the individual loops stable, which in most cases is an advantage. However, integral action is inherent in IMC controllers, and integral action and stability of the individual loops are 
known to be incompatible with stability of the overall system for certain plants. The Niederlinski index criterion (1971) gives a necessary condition for obtaining stability both of the individual loops and the overall system when there is integral action in all channels. The Niederlinski index criterion has recently been generalized to open loop unstable plants (Hovd and Skogestad 1992b). Let the number of right half plane (RHP) poles in $G$ be $n_{\mathrm{U}}$ (including multiplicities), and the number of RHP poles in $\tilde{G}$ be $\tilde{n}_{\mathrm{U}}$. Note that in general $\tilde{n}_{\mathrm{U}} \neq n_{\mathrm{U}}$. If all the individual loops are stable, a necessary condition for the stability of the overall system is that the sign of the Niederlinski index, $N_{\mathrm{I}}=\operatorname{det} G(0) / \operatorname{det} \tilde{G}(0)$, is positive if $\tilde{n}_{\mathrm{U}}-n_{\mathrm{U}}$ is even and negative if $\tilde{n}_{\mathrm{U}}-n_{\mathrm{U}}$ is odd. Thus, before attempting to perform an independent design, one should check that overall stability can be achieved with integral action in all channels and having stable individual loops.

Example 3. We would like to emphasize Step 4 in the independent design procedure, that nominal stability must be checked explicitly for one value of $\epsilon_{i}$ within the bounds found. Consider the process:

$$
\boldsymbol{G}(s)=\left[\begin{array}{cc}
\frac{5}{20 s^{2}+12 s+1} & \frac{8}{20 s^{2}+12 s+1} \\
\frac{6}{40 s^{2}+12 s+1} & \frac{2}{40 s^{2}+12 s+1}
\end{array}\right]
$$

with independent actuator uncertainty with uncertainty weight $W_{\mathrm{I}}((s)=0 \cdot 2((10 s+1) /$ $(s+1)) I_{2}$. Since this plant is stable we have that $\tilde{n}_{\mathrm{U}}-n_{\mathrm{U}}=0$ and since the Niederlinski index is negative, $N_{1}=-3 \cdot 8$, we know that we cannot have the individual loops stable and at the same time achieve overall system stability. Nevertheless, assume that we proceed with independent design, and choose third-order low-pass filters for both loops. We find that Step 2 in the independent design procedure indicates that any value of $c>4$ (approximately) will give robust stability (figure omitted). Calculating $\mu$ for $\epsilon=5$ for both loops, we do indeed obtain a value of $\mu<1$ at all frequencies. The reason, which we find in Step 4 in the independent design procedure, is that the overall system is nominally unstable. The $\mu$ test merely tells us that this instability is a robust property. For other cases, it may not be this easy to tell a priori that the overall system will be unstable with the individual loops stable.

\section{Discussion}

For both Examples 1 and 2, Chiu and Arkun (1992) and Chiu (1991) were unable to perform an independent design, using the procedure of Skogestad and Morari (1989). We have shown that a solution is possible using the proposed independent IMC design procedure. This demonstrates the importance of introducing as little conservatism as possible in the description of the design uncertainty associated with the controllers when performing an independent design.

The bounds obtained in the proposed independent IMC design procedure are common to all the filter elements, and it is not obvious how to take advantage of the possibility of having differing filter time constants in the different filter elements. Trying to add a performance requirement to Example 2 illustrates the difficulty with applying independent design to problems for which different bandwidths in the different loops are necessary. One may use constant ratios between the filter time constants in our independent design procedure (e.g. choosing $\epsilon_{1}=\epsilon^{*}, \epsilon_{2}=10 \epsilon^{*}$ etc.) but in this case a simple parameter search for $c^{*}$ and $e^{*}$ would be much simpler. 
One can relatively easily use parameter optimization to find the IMC filter time constants $\epsilon_{i}$ that minimize $\mu(M)$, and a valid question is whether this is a better approach. The answer is that although our independent design procedure cannot be used to find the optimal filter time constants, it has the advantage of providing a range of values for which robust stability/performance is fulfilled, and can also guarantee that the system is robust decentralized detunable. For example, applying parameter optimization to Example 1 we find that the optimal value of $\mu$ is 0.60 , which is achieved for $c_{1}=0.10$ and $c_{2}=7.67$. However, parameter optimization gives no information about how robust performance deteriorates when the filter time constants are changed from their optimal values, and cannot show that the robust performance requirements are fulfilled for any $\epsilon_{i}$ in the range $3 \cdot 7<\epsilon_{i}<6 \cdot 6$. Furthermore, parameter optimization cannot be used to find that the system is robust decentralized detunable provided $\epsilon_{i}>0 \cdot 16$.

\section{Conclusions}

We have proposed a parameterization of the class of allowable decentralized designs which is based on the following four key steps:

(1) Use an IMC controller design for each loop.

(2) Select the filter time constant $\varepsilon_{i}$ as the 'uncertain' parameter.

(3) Parametrize $\epsilon_{i}$ and $e_{i}=1 / \epsilon_{i}$ such that only positive values are allowed.

(4) Obtain bounds on both $\epsilon_{i}$ and $e_{i}$ that guarantee robust stability/performance.

A new independent design procedure based on the above parameterization is presented and we have found that:

- The result of considering only decentralized IMC controllers with a specified filter structure is that the set of possible controller designs considered is much smaller than the set of possible controller designs when trying to find bounds on $\tilde{\boldsymbol{S}}$ and $\tilde{\boldsymbol{H}}$, and the resulting bounds are therefore less conservative.

- One can derive a bound on the IMC filter time constants which ensures that the system is robust decentralized detunable.

- It is critical that real perturbations are used for the parameterizations of $\epsilon_{i}$ and $e_{i}$. $\mu$ software capable of handling real perturbations is therefore needed.

- The procedure is difficult to apply for problems where different bandwidths in the various loops are desired.

The independent design procedure proposed here can also be applied to other types of controllers. For example, one can find bounds on the ratio of gain to integral time $\left(k / \tau_{1}\right)$ for PID controllers. We can only find bounds for one parameter at the time; the other parameters have to be found by some other method in advance. In contrast, decentralized IMC controllers have only one tuning parameter, and are therefore preferable for our independent design procedure.

\section{ACKNOWLEDGEMENT}

The authors wish to thank M. P. Newlin and P. M. Young of California Institute of Technology for access to their software for calculating $\mu$ with mixed real and complex perturbations. Support from the NTNF is gratefully acknowledged.

$\dagger$ After restricting $\epsilon_{i} \geqslant 0 \cdot 10 i=1,2$, in the same way as in Reference 3 . 


\section{REFERENCES}

Braftz, R. D., Morari, M. and Skogestad, S. (1992). AlChE Annual Meeting Miami Beach, Florida, paper 127 a, preprint, 1992.

CHIU, M.-S. (1991). A Methodology for the Synthesis of Robust Decentralized Control Systems. PhD Thesis, Georgia Institute of Technology, Atlanta, GA, USA, 1991.

ChIU, M.-S. and Arkun, Y. (1992). Automatica, 28, 997.

Doyle, J. C. and ChU, C.-C. (1985). Proc. American Control Conference,' Boston, MA, 1985, p. 129.

Doyle, J. C., Wall, J. E. and Stein, G. (1982). Proc. IEEE Conf. Decision Contr.,' Orlando, FL., 1982, p. 629.

Garcia, C. E. and Morari, M. Ind. Eng. Chem. Proc. Des. Dev., 1982, 21, 308.

Hovd, M. and Skogestad, S. (1992a). Automatica, 28, 989; (1992b). Proc. IF AC Workshop on Interactions between Process Design and Control, London, September 1992; (1992c). AIChE Annual Meeting, Miami Beach, Florida, paper 127d, 1992.

LEE, J. H. and Morari, M. (1991). Automatica, 27, 519.

MACIEJOWSKı, J. M. (1989). Multivariable Feedback Design (Addison-Wesley, Wokingham).

MAYNE, D. Q. (1973). Automatica, 9, 201.

Nett, C. N. and UthgenanNt, J. A. (1988). Automatica, 24, 261.

NiEDERLINSKI, A. (1971). Automatica, 7, 691.

Skogestad, S., and Lundström, P. (1990). Comput. Chem. Engng. 14, (4/5), 401

Skogestad, S. and Morari, M. (1988). IEEE Trans. Autom. Control, 33, 1151; (1989). Automatica, 25, 119.

Young, P. M., Newlin, M. P. and Doyle, J. C. (1991). 'Proc. 30th CDC,' Brighton, UK, 1991, 1251. 